\title{
Postural responses of galvanic vestibular stimulation: comparison between groups of older adults and young people
}

Abstract

Objective: To evaluate the effect of vestibular manipulation on the postural sway and muscle activation of younger and older adults. Methods: The study analyzed the effects of three intensity levels of galvanic vestibular stimulation (GVS) $(0.3 ; 0.6$ and $1 \mathrm{~m})$ on the pattern of muscle activity and center of pressure (CP) displacements of 12 older adults (EG) and 12 young adults (CG) while maintaining their balance on a stable surface, with no vision. Results: The EG showed a positive correlation between CP displacement and muscle activity and GVS intensity. On the other hand, the magnitude of postural response in the EG was not modulated in accordance with GVS intensities. Additionally, during the highest GVS intensity level $(1 \mathrm{~mA})$ greater muscle activity was used to increase stiffness, decrease the amplitude of oscillation and ensure stability. This unusual response characterizes a pattern of co-activation and is perhaps a safety mechanism to ensure stability. Conclusion: The EG individuals were not able to select the appropriate motor strategy to efficiently compensate the effects of GVS. This unusual strategy reflects deficits in the vestibular system of older adults, a fact which negatively interferes with their ability to reevaluate sensory information.

\footnotetext{
Centro Universitário das Faculdades Associadas de Ensino (FAE), Departamento de Fisioterapia. São João da Boa Vista, SP, Brasil.

2 Universidade de São Paulo, Faculdade de Medicina. Ribeirão Preto, SP, Brasil.

3 Universidade de São Paulo, Faculdade de Medicina, Departamento de Biomecânica, Medicina e Reabilitação do Sistema Locomotor. Ribeirão Preto, SP, Brasil.

Funding: Fundação de Amparo à Pesquisa de São Paulo (FAPESP - no 2007 / 54596-0; nº 07 / 07606-0).

The authors declare there are no conflicts of interest in relation to the present study.

Keywords: Postural Balance. Vestibule Labyrinth. Health of the Elderly. 


\section{INTRODUCTION}

Postural control is performed by motor and sensory mechanisms ranging from relatively simple peripheral responses to complex events involving high levels of cognitive function and sensorimotor integration. With the aging process, there is a decline in motor and sensory functions, impairing postural stability and increasing the number of falls. Falls are a major public health problem in the older population. As people age, the incidences of falls increases. According to the World Health Organization, between $28-35 \%$ of people aged 65 and over fall each year and this number rises to $32-42 \%$ from the age of $70^{1}$.The resulting injuries can lead to reduced levels of independence, poor quality of life, and high levels of anxiety ${ }^{1}$.

It is therefore vital that any risk factors associated with instability and falling are identified early. Early detection of these factors can guide preventive programs, making them more efficient and less costly. However, several risk factors contribute to postural instability, and these can impair the detection of specific causes of a decline in instability.

Possible explanations for the increased risk of falls include cognitive limitations ${ }^{2}$, musculoskeletal changes such as reduced bone mineral density ${ }^{3}$, a reduction in the number of muscle fibers, which alter muscle length changes and reduce strength ${ }^{4}$, a decline in knee and ankle joint torque ${ }^{5,6}$, slower muscle response after an external disturbance ${ }^{5,7}$, sensory changes such as a reduction in spatial perception ${ }^{8}$, compromised vestibular information ${ }^{9}$, reduced visual acuity and less sensitivity to contrasts ${ }^{10}$, reduced skin sensation ${ }^{11}$ and proprioceptive changes ${ }^{12}$.

There is no consensus about which system (sensory, motor or cognitive) exerts the greatest influence over the decline of postural control among older adults. Krager ${ }^{13}$ suggests that changes in the vestibular system are an important contributor to such a decline. Other studies that support the idea of decline of the vestibular system are those that describe the anatomical changes resulting from aging, among which the loss of sensory hair cells ${ }^{14,15}$ and vestibular nucleus neurons ${ }^{16}$ are of particular relevance.
The role of the vestibular system in postural control can be studied by artificially manipulating the vestibular afferences by applying galvanic current (continuous bipolar) to mastoid processes by depolarizing the vestibular nerve and increasing the triggering frequency of vestibular afferents on the cathode side, while decreasing the afferents on the anode side ${ }^{9}$. Such application results in body sway to the anode side. This behavior is similar to that observed in individuals with vestibular system disorders, and allows a more specific assessment of vestibular information by inducing postural reactions during static posture maintenance. It is therefore a useful way of studying the role of vestibular information in the postural control of older adults ${ }^{13,17}$.

Considering the decline of sensory information as a relevant factor in the postural control of older adults, as well as the fact that this control depends on a complex integration of multiple sensory systems (including the vestibular system), the study of the affect of three intensities of galvanic vestibular stimulation (GVS) on the postural oscillation of the older adults was proposed.

\section{METHOD}

A quantitative cross-sectional study was carried out with a sample of 12 older adults (EG) and 12 younger adults (CG) resident in the community of Ribeirão Preto, São Paulo, Brazil. The evaluated group was selected from unintentional convenience sampling, based on participation in social groups for the EG and from the university for the CG, due to the difficulty of random selection.

Participants were invited to participate voluntarily. All were informed of the objectives and procedures of the study and were asked to sign a Consent Form approved by the research ethics committee: CAA 0245.0.213,000-10.

The population sample consisted of the following inclusion criteria: aged 65 years or older for the EG and 18 for the CG; be able to walk without an assistive device and have a MMSE score above 22 points. 
Individuals with mobility deficits (unable to walk for six meters independently), unstable or severe health conditions, such as strokes, Parkinson's disease, Alzheimer's disease, epilepsy, who used pacemakers, had suffered neuropathies, had previously suffered vestibulopathies and who were unable to understand the simple instructions necessary for performing the required tasks were excluded.

The individuals went to the Balance Evaluation and Rehabilitation Laboratory (or LARE) of the Faculty of Medicine of Ribeirão Preto (FMRP USP) where data collection was performed during a session with a duration of approximately $90 \mathrm{~min}$.

To evaluate cognitive function, history of dizziness, functional mobility and activities of daily living, the Mini Mental State Examination (MMSA), Performance Oriented Mobility Assessment $(\text { POMA })^{19}$, Dizziness Handicap Inventory (DHI) ${ }^{20}$ and the Lawton Instrumental Activities of Daily Living scale (IADL) ${ }^{21}$ were administered.

The subjects were required to maintain a motionless stance for 10 s while barefoot on a force plate and under four different conditions (Chart 1). The center of pressure (CP) displacement and electromyographic (EMG) activities of certain postural muscles were recorded. The investigator ensured that the feet of the subjects were arranged on the center of the force plate. To control for prediction, habituation and fatigue, the four conditions were randomized and repeated three times for each individual, totaling 12 trials per subject.

Chart 1. Tasks analyzed during the maintenance of static balance on a force platform. Ribeirão Preto, SP, 2017

\begin{tabular}{|l|l|}
\hline Tasks & Sensorial Stimulation \\
\hline 1 & Eyes closed without stimulation \\
\hline 2 & Eyes closed with GVS of $0.3 \mathrm{~mA}$ \\
\hline 3 & Eyes closed with GVS of $0.6 \mathrm{~mA}$ \\
\hline 4 & Eyes closed with GVS of $1.0 \mathrm{~mA}$ \\
\hline
\end{tabular}

GVS: Galvanic vestibular stimulation

The bipolar and binaural transmastoid current was randomly applied via $3.5 \mathrm{~cm}^{2}$ carbon-rubber electrodes placed behind the ears of the subjects over the mastoid processes. The anode was placed on the right ear. The intensities applied were 0.3 , 0.6 and $1 \mathrm{~mA}$.

\section{Data analysis}

Posture sway was quantified using the $\mathrm{CP}$ while standing on a force platform covering an area of $50 \times 50 \mathrm{~cm}^{2}$ (model Biomec400, EMG System Brazil). This force platform was composed of four load cells at each corner, and the vertical load applied to each cells was measured. These loads were simultaneously acquired at a $100 \mathrm{~Hz}$ sampling frequency with a 12-bit resolution A/D card. From these signals, the resulting vertical ground reaction force and
$\mathrm{CP}$ position in both the anterior-posterior and mediolateral directions were calculated. The true location of the CP at this force plate had a mean error of $0.02 \mathrm{~cm}$ from the factory calibration. The CP signals were filtered through a low-pass, fourth-order Butterworth filter with zero-lag at a cut-off frequency of $10 \mathrm{~Hz}$. To quantify body sway, the amplitude and standard deviation (SD) of CP displacement were calculated in the respective directions.

The electromyographic (EMG) activity of the gastrocnemius medialis (GM), tibialis anterior (TA), biceps femoris (BF) and rectus femoris (RF) muscles were recorded using electromyography (EMG System do Brasil). Self-adhesive $\mathrm{Ag} / \mathrm{AgCl}$ bipolar surface electrodes were used at an inter-electrode distance of $2 \mathrm{~cm}$. The electrodes were connected to a 16-channel signal conditioner (EMG Systems Brazil). The electromyography signals of the muscles 
were digitally recorded using an A/D converter board with a 16-bit dynamic resolution. The sampling frequency was set at $2 \mathrm{KHz}$ with an input range of $\pm 10 \mathrm{~V}$. The electromyography signal was filtered through a Butterworth pass-band filter $(20-500 \mathrm{~Hz})$

The root mean squares (RMS) of the four muscle activities cited above were calculated for each trial, and the values were normalized by maximal voluntary contraction.

\section{Statistical analysis}

Data analysis employed descriptive statistics. For the quantitative variables, measures of central tendency (mean) and dispersion (standard deviation) were calculated. For values measured in an ordinal scale (MMSE, POMA, DHI), it was decided to use a nonparametric test for independent samples, namely the Mann-Whitney U test for comparison between groups.

Analysis of variance (ANOVA) was used to verify if the GVS intensities $(0,0.3,0.6$ and $1 \mathrm{~mA})$. had different effects on the activation (RMS value) of each evaluated muscle. Considering the objective of evaluating the effect of intensity on muscle activation, intra-group analysis was selected.
For the comparison between the groups, the values of antero-posterior and mediolateral center of pressure displacement were used, and the displacement in each direction was analyzed by ANOVA with two factors: stimulation $x$ group. The significance level was set at 0.05 .

The relationship between CP displacement $\mathrm{x}$ GVS intensities and muscle activation (RMS) $\mathrm{x}$ GVS intensities was calculated using Pearson's linear correlation coefficient.

\section{RESULTS}

A total of 24 individuals divided into two groups participated in the study. The older adults group (EG) composed of 12 volunteers $(75 \pm 8.86$ years, eight women and four men, BMI of 26.21 \pm 4.86 ) living in the community of Ribeirão Preto; and the Control Group (CG) composed of 12 adults $(23 \pm 5$ years nine women and three men, BMI 24.32 \pm 4.20 ), who were undergraduate students of the physiotherapy course at USP.

There was no significant difference between the groups on the Lawton scale. The CG achieved the maximum score of 27 , with a similar performance among the EG (Table 1).

Table 1. Scores of Lawton Scale, MMSE, DHI e POMA in the control group ( $\mathrm{n}=12)$ and older adult group $(\mathrm{n}=12)$. Ribeirão Preto, São Paulo, 2017.

\begin{tabular}{llll}
\hline Instruments & Groups & Mean (standard deviation) & $p$-value \\
\hline Lawton & CG & $27( \pm 0)$ & 0.17 \\
& EG & $26.3( \pm 2.3)$ & $0.02^{*}$ \\
\hline MMSE & CG & $29( \pm 1.3)$ & 0.16 \\
& EG & $26.16( \pm 2.7)$ & $0.00^{*}$ \\
\hline PHI & CG & $0.83( \pm 8.5)$ & $2.33( \pm 5.5)$ \\
\hline
\end{tabular}

Lawton=Instrumental Activities of Daily Living Scale, MMSE=Mini Mental State Examination, DHI=Dizziness Handicap Inventory, POMA $=$ Performance Oriented Mobility Assessment; ${ }^{*} p<0,05$. 
There were no statistical differences in DHI between the two groups, which was expected as vestibular disorders were an exclusion criteria. DHI assesses possible vestibular changes in three dimensions: physical, emotional and functional. The worst performance score observed in EG individuals was 100 points, a value that did not indicate vestibular deficits.

In contrast, a significantly different effect was observed between the groups in the MMSE and the POMA. Notably, however, the values obtained by the older adults in both the MMSE (over 27 points) and POMA (over 46 points) do not indicate cognitive or mobility impairments (Table 1).

\section{EMG Activity}

The analysis of muscle activity during the maintenance of balance, with or without the three intensities of GVS, indicated that for the EG, the higher the stimulus intensity, the greater the muscle activation. Thus, with increased magnitude, the muscles responded in a linear fashion (as can be seen from the correlation coefficient values above 0.9 presented in Figure 1). The 9\% increase in TA muscle activity during $0.3 \mathrm{~mA}$ GVS in relation to the activity without stimulus (12\% during $0.6 \mathrm{~mA}$ and $27 \%$ at a current of $1 \mathrm{~mA}$ ) is notable. Other muscles demonstrated similar behavior. The GM increased by $10 \%, 16 \%$ and $47 \%$; the BF increased by $13 \%, 20 \%$ and $35 \%$; and the RF increased by $20 \%, 22 \%$ and $25 \%$, at intensities of $0.3,0.6$ and $1 \mathrm{~mA}$, respectively.
The muscle activity of the EG also increased with GVS, but not in the same linear fashion (Figure 1). There was a decrease in muscle activity of $4 \%$ for the TA, $6 \%$ for the GM, 3\% for the BF and $0,5 \%$ for the RF at $0.3 \mathrm{~mA} \mathrm{GVS}$, compared to activity without stimulus. At $0.6 \mathrm{~mA}$ of GVS, the activities of the TA and GM were similar to their activities at $0.3 \mathrm{~mA}$, while the BF decreased by $5 \%$ and the RF decreased by $7 \%$. At $1 \mathrm{~mA}$ of GVS, the activity of all muscles increased: $43 \%$ for the TA, $54 \%$ for the GM, $37 \%$ for the BF and $42 \%$ for the RF. For the EG, muscle activity increased primarily at higher GVS intensity levels.

For the CG, ANOVA testing showed no intensity effect for the TA ( $\mathrm{F}=2.25 ; \mathrm{P}=0.14), \mathrm{RF}(\mathrm{F}=2.10$; $\mathrm{P}=0.16), \mathrm{GM}(\mathrm{F}=15.47 ; \mathrm{P}=0.04)$ and $\mathrm{BF}(\mathrm{F}=7.10$; $\mathrm{P}=0.013)$ muscles. For the EG, ANOVA testing showed no intensity effects for the previously mentioned muscles.

\section{CP displacement}

It was note that in the CG, a positive correlation between GVS intensity and postural oscillation was observed when evaluated by amplitude (Figure 2) and $\mathrm{SD}$ (Figure 3) of $\mathrm{CP}$ in an anterior to posterior (AP) direction. The correlation coefficient values are greater than nine. Although the medial to lateral (ML) displacement also increased with GVS intensity, linear behavior was less evident in this direction. 


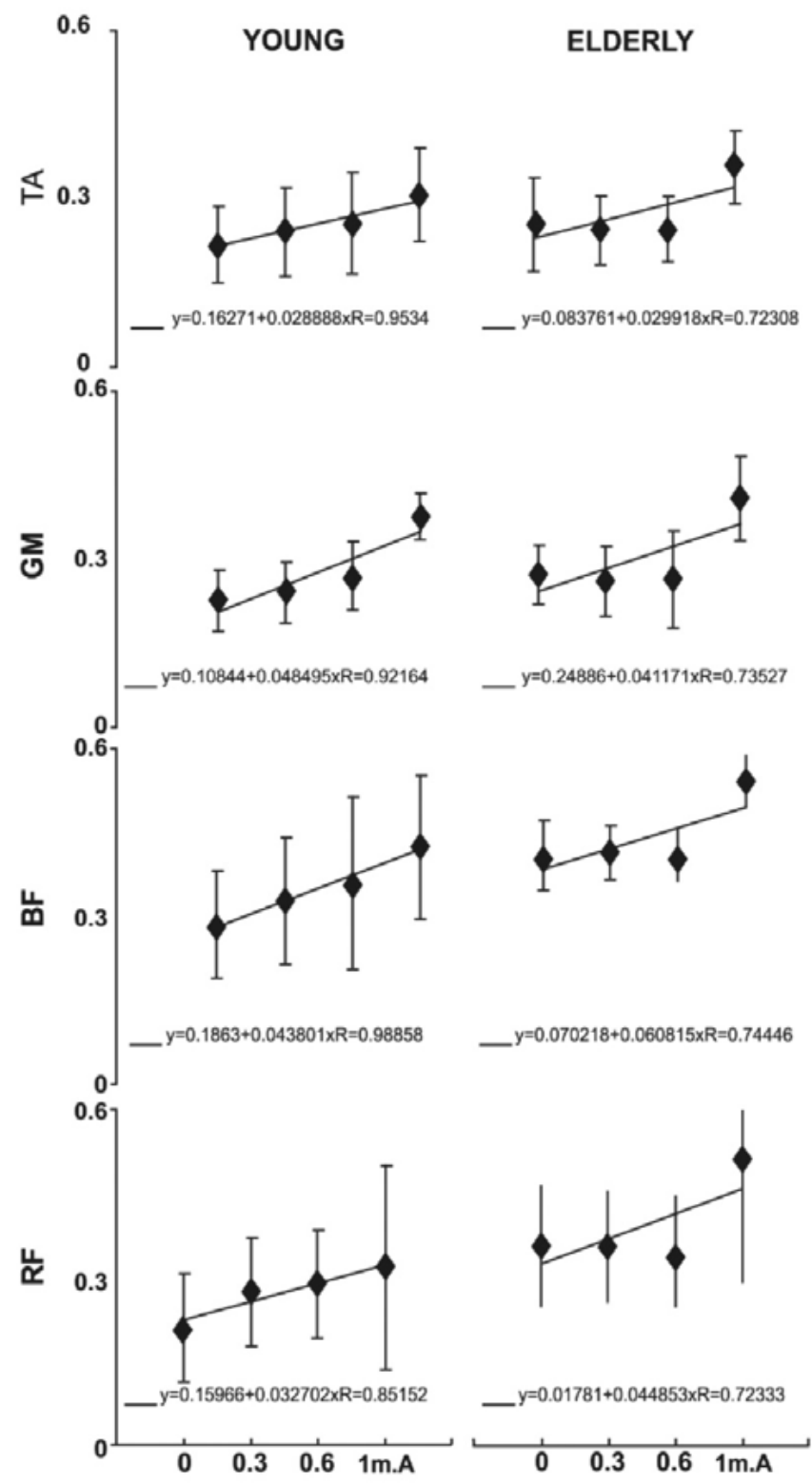

Figure 1. EMG activity of the tibialis anterior (TA), gastrocnemius medialis $(\mathrm{GM})$, biceps femoris $(\mathrm{BF})$ and rectus femoris $(\mathrm{RF})$ muscles during upright balance analysis on the force platform associated with different intensities of galvanic vestibular stimulation $(0,0.3,0.6$ and $1 \mathrm{~mA})$ in the younger adults (Control Group) and older adults groups. Ribeirão Preto, São Paulo, Brazil, 2017. 
ML

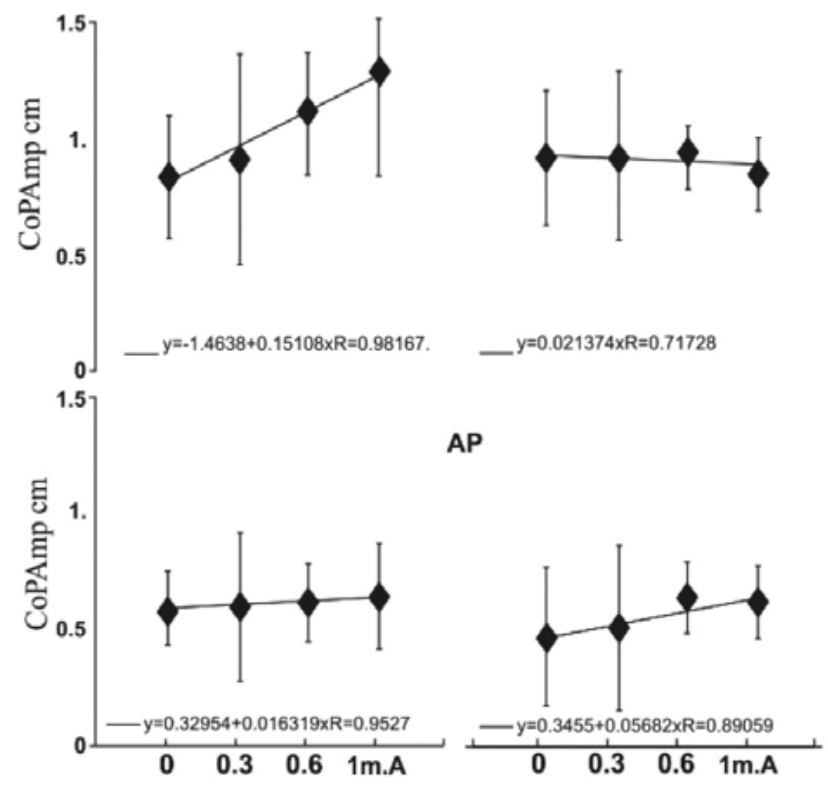

Figure 2. Amplitude for the displacement of $\mathrm{CP}(\mathrm{cm})$ in medial to lateral direction (ML) (upper panel) and anterior to posterior direction (AP) (lower panel) during different galvanic vestibular stimulation (GVS) intensities (0, 0.3, 0.6 and $1 \mathrm{~mA}$ ) for the CG (left panel) and EG (right panel). Correlations between GVS intensities and Amplitude of CP displacement were also presented. Ribeirão Preto, São Paulo, Brazil, 2017.

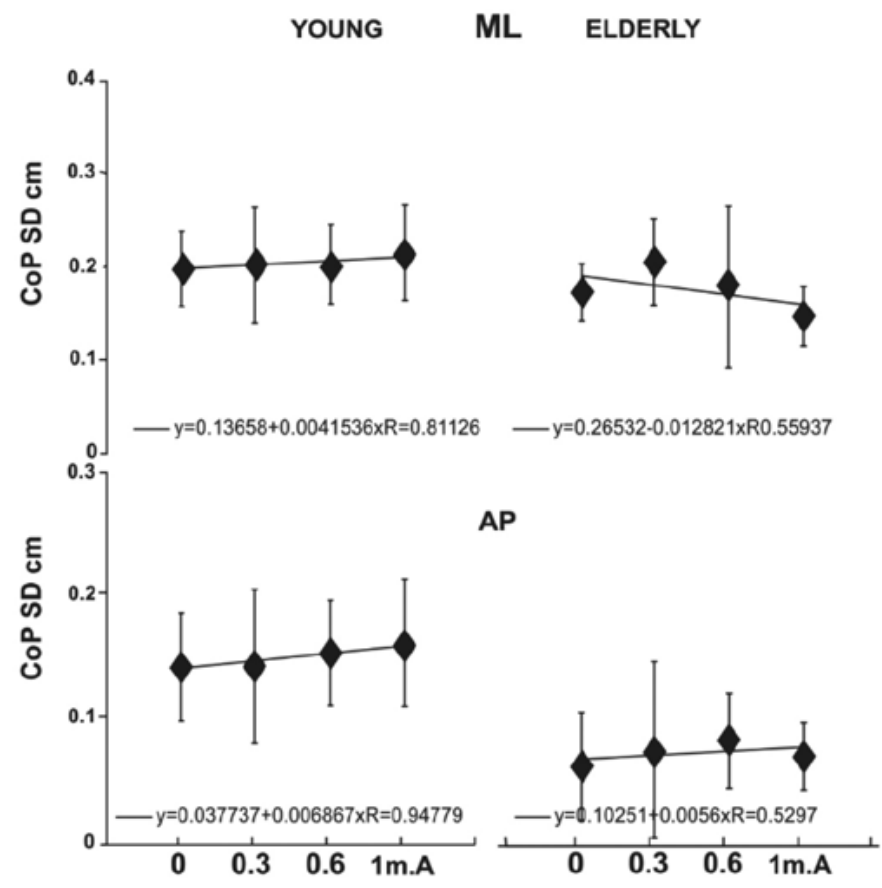

Figure 3. Standard deviation for the displacement of CP $(\mathrm{cm})$ in medial to lateral direction (ML) (upper panel) and anterior to posterior direction (AP) (lower panel) during different galvanic vestibular stimulation (GVS) intensities $(0,0.3,0.6$ and $1 \mathrm{~mA})$ for the younger adults (CG) and older adults (EG) groups. Ribeirão Preto, São Paulo, Brazil 2017. 
The EG were not able to modulate any postural oscillation with the GVS intensity (see the low values of correlation coefficients in Figures 2 and 3). They also showed a minor postural oscillation towards the AP direction during the 3 intensities of GVS, compared to the CG (amplitude $\mathrm{F}=0.00 ; \mathrm{P}=0.04$ ) $(\mathrm{SD} \mathrm{F}=9.60 \mathrm{P}=0.03)$.

\section{DISCUSSION}

The present study compared postural sway and EMG muscle activities in a motionless stance between older and younger adults. In addition, the correlation between postural sway and the magnitude of GVS stimulation was measured. The primary findings for the CG were that (1) there was a significant increase with GVS in the GM and BF muscle activity, and (2) there was a positive correlation between postural response and GVS intensity. The main findings for the EG were that (3) these individuals were not able to modulate the magnitude of postural response with the intensities of GVS stimulus, and (4) they responded only to high levels of GVS intensity, indicating a possible deficit in vestibular information processing.

Increasing the intensity of GVS among the CG also increased the anterior postural response. This finding corroborates the studies of Horak \& Hlavacka $^{22}$, who observed a proportional increase in body displacement with increasing intensity levels of GVS. This observation demonstrates the ability of the vestibular system to differentiate between graded levels of information and contributes to an altered response in magnitude.

The intensity required to trigger postural sway in the CG was similar to that found by Lee et al. ${ }^{23}$; Hlavacka et al. ${ }^{24}$ and Inglis et al. ${ }^{25}$, who reported an intensity level of 0.2 to $0.5 \mathrm{~mA}$. In addition, Rinalduzzi et al. ${ }^{26}$, demonstrated that even at $0.7 \mathrm{~mA}$, GVS modulates neuronal excitability and produces recordable vestibular body sway in healthy subjects.

The effect of GVS observed in the AP direction is in agreement with literature, which demonstrates that bilateral stimuli induce increased oscillation by modulating the rate of triggering vestibular nerves ${ }^{26}$. Sway is influenced according to head position (in the present study turned to the right), towards the side of the anodal electrode, by modulating the firing rate of the vestibular nerves. ${ }^{26}$ Taking these concepts together with the head position adopted in the present study, it is possible to justify the significant effect of GVS only on the posterior muscles (i.e., the GM and BF muscle groups).

The most important findings relate to the inability of the EG to modulate their postural responses with GVS intensity and that they respond only to higher levels of GVS intensity. Although the effects of GVS on the older adults are less explored, several studies have evaluated vestibular function through vestibular evoked myogenic potential (VEMP) ${ }^{27}$ The effect of age on VEMP amplitude is well established, and numerous studies have determined the decrement in amplitude among individuals older than 60 years ${ }^{1,28}$. Although the techniques are different, the outcomes between studies of VEMP and GVS are similar, indicating a decreased response with age. This decrement can be attributed to age-related changes in the vestibular system, such as the loss of sensory hair cells ${ }^{14}$ and the degeneration of vestibular nerve fibers in the Scarpa's ganglion cells ${ }^{15}$ and the vestibular nucleus neurons ${ }^{16}$

Aoyamak et al. ${ }^{26}$ found a lower postural response to a GVS level of $0.7 \mathrm{~mA}$ in individuals with vestibular disorders. They justified this finding by claiming that GVS was unable to modulate the function of the vestibular system as it would already be impaired by dysfunction. In this sense, the lower response to GVS observed at low intensities in the older adults in the study could be explained by the presence of dysfunction in this system.

However, with a greater level of GVS intensity $(1 \mathrm{~mA})$, an increase in muscle activity was observed that increased stiffness, decreased the amplitude of oscillation and ensured stability in the EG. These unusual responses characterize a pattern of coactivation and may be a safety strategy assumed to ensure stability due to sensorial reorganization impairments in the older adults.

It is well established that older adults undergo somatosensory changes, which according to some authors, are responsible for a decline in postural control $^{5,29,30}$. It is also known that individuals with somatosensory deficits increase the sensitivity of 
vestibular information and consequently increase their response to GVS. Therefore, one would expect that due to somatosensory deficits, the response of older adults to GVS will increase. This behavior occurred only during greater stimulation $(1 \mathrm{~mA})$, and even then, the postural response was atypical. This finding supports the idea that when the vestibular system of older adults is compromised, it negatively interferes with their ability to reevaluate sensory information.

It is important to highlight certain limitations of the present study, in relation to the non-randomness of the sample selection and the sample size. This fact prevents possible generalizations for the Brazilian population, and the results should be considered as preliminary. In terms of gerontological care, the results suggest that multiprofessional health teams should be aware of the presence of sensory processing disorders and their impacts on postural control in older adults.

\section{REFERENCES}

1. Commandeur D, Klimstra MD, MacDonald S, Inouye $\mathrm{K}$, Cox M, Chan D, et al. Difference scores between single-task and dual task gait measures are better than clinical measures for detection of fall-risk in community-dwelling older adults. Gait Posture. 2018;66:155-9.

2. Jehu DAM, Paquet N, Lajoie Y. Balance and mobility training with or without simultaneous cognitive training reduces attention demand but does not improve obstacle clearance in older adults. Motor Control. 2018;22:275-94.

3. Miko I, Szerb I, Szerb A, Bender T, Poor G. Effect of a balance-training programme on postural balance, aerobic capacity and frequency of falls in women with osteoporosis: a randomized controlled trial. J Rehabil Med. 2018;50(6):542-7.

4. Hernández-Luis R, Martín-Ponce E, MonereoMuñoz M, Quintero-Platt G, Odeh-Santana S, González- Reimers E, et al. Prognostic value of physical function tests and muscle mass in elderly hospitalized patients: a prospective observational study. Geriatr Gerontol Int. 2018;18:57-64.

5. Crenshaw JR, Bernhardt KA, Atkinson EJ, Khosla S, Kaufman KR, Amin S. The relationships between compensatory stepping thresholds and measures of gait, standing postural control, strength, and balance confidence in older women. Gait Posture. 2018;65:74-80.

\section{CONCLUSION}

The EG individuals were unable to select the appropriate motor strategy to efficiently compensate the effects of GVS. They responded only to high intensity GVS, indicating the alteration of the vestibular system response threshold. This atypical strategy may reflect deficits in the vestibular system and negatively affect the ability to reorganize sensory information, impairing the efficiency of postural responses when faced with sensory information inaccuracy. Guidelines should be given to older adults in relation to the risk of imbalance in relation to sensory conflicts, and sensory manipulation should be considered as an auxiliary resource in rehabilitation programs aimed at improving the postural control of older adults.

Edited by: Tamires Carneiro Oliveira Mendes

6. Currie DW, Thoreson SR, Clark L, Goss CW, Marosits MJ, Diguiseppi CG. Factors associated with older adults enrollment in balance classes to prevent falls: case-control study. J Appl Gerontol. 2018;18:73-80.

7. Shulman D, Spencer A, Vallis LA. Age-related alterations in reactive stepping following unexpected mediolateral perturbations during gait initiation. Gait Posture. 2018;64:130-4.

8. Shaffer SW, Harrison AL. Aging of the somatosensory system: a translational perspective. Phys Ther. 2007;87(2):193-207.

9. Horak FB, Kluzik J, Hlavacka F. Velocity dependence of vestibular information for postural control on tilting surfaces. J Neurophysiol. 2016;116:1468-79.

10. Saftari LN, Kwon OS. Ageing vision and falls: a review. J Physiol Anthropol. 2018;37:23-37.

11. Woo MT, Davids K, Liukkonen J, Chow JY, Jaakkola T. Immediate effects of wearing knee length socks differing in compression level on postural regulation in community-dwelling, healthy, elderly men and women. Gait Posture. 2018;66:63-9.

12. Paton J, Glasser S, Collings R, Marsden J. Getting the right balance: insole design alters the static balance of people with diabetes and neuropathy. J Foot Ankle Res. 2016;9:5-9. 
13. Krager R. Assessment of vestibular function in elderly patients. Curr Opin Otolaryngol Head Neck Surg. 2018;26(5):302-6.

14. Taylor RR, Jagger DJ, Saeed SR, Axon P, Donnelly $\mathrm{N}$, Tysome J, et al. A. Characterizing human vestibular sensory epithelia for experimental studies: new hair bundles on old tissue and implications for therapeutic interventions in ageing. Neurobiol Aging. 2015;36:2068-84.

15. Zu Eulenburg P, Ruehl RM, Runge P, Dieterich. Ageing-related changes in the cortical processing of otolith information in humans. Eur J Neurosci. 2017;46:2817-25.

16. Gluth MB, Nelson EG. Age-related change in vestibular ganglion cell populations in individuals with presbycusis and normal hearing. Otol Neurotol. 2017;38:540-6.

17. Lui KY, Hewston P, Deshpande N. VisualVestibular interaction for postural control during Sit-to-Stand: effects of aging. Motor Control. 2018;14:1-12.

18. Dag E, Örnek N, Örnek K, Günay F, Türkel Y. Mini mental state exam versus Montreal cognitive assessment in patients with age-related macular degeneration. Eur Rev Med Pharmacol Sci. 2014;18(20):3025-8.

19. Knobe M, Giesen M, Plate S, Gradl-Dietsch G, Buecking B, Eschbach D, et al.The Aachen Mobility and Balance Index to measure physiological falls risk: a comparison with the Tinetti POMA Scale. Eur J Trauma Emerg Surg. 2016;42(5):537-45.

20. Mutlu B, Serbetcioglu B. Discussion of the dizziness handicap inventory. J Vestib Res. 2013;23(6):271-7.
21. Kadar M, Ibrahim S, Razaob NA, Chai SC, Harun D. Validity and reliability of a Malay version of the Lawton instrumental activities of daily living scale among the Malay speaking elderly in Malaysia. Aust Occup Ther J. 2018;65(1):63-8.

22. Horak FB, Hlavacka F. Somatosensory loss increases vestibulospinal sensitivity. J Neurophysiol. 2001;86:575-85.

23. Jeong-Woo Lee, Woong-Sik Park, Se-Won Yoon. Effects of galvanic vestibular stimulation on event related potentials. J Phys Ther Sci. 2016;28:2604-7.

24. Hlavack F, Shupert LC, Horak FB. The timing of galvanic vestibular stimulation affects responses to platform translation. Brain Res. 1999;821:8-16.

25. Čobeljić R, Miljković N, Ribarić-Jankes k, Švirtlih L. A paradigm of galvanic vestibular stimulation diminishes the soleus muscle H-reflex in healthy volunteers. Spinal Cord. 2015;54:1-4.

26. Aoyamak, Lizuka H, Ando H. Four-pole galvanic vestibular stimulation causes body sway about three axes. Sci Rep. 2015;(5):101-68.

27. Zu Eulenburg P, Ruehl RM, Runge P, Dieterich M. Ageing-related changes in the cortical processing of otolith information in humans. Eur J Neurosci. 2017;46:2817-25.

28. Brantberg K, Granath K, Schart N. Age-related changes in vestibular evoked myogenic potentials. Audiol Neurootol. 2007;12(4):247-53.

29. Lord SR, Menz HB. Visual contributions to postural stability in older adults. Gerontology. 2000;46(6):306-10.

30. Brown KE, Neva JL, Feldman SJ, Staines WR, Boyd LA. Sensorimotor integration in healthy aging: Baseline differences and response to sensory training. Exp Gerontol. 2018;112:1-8. 